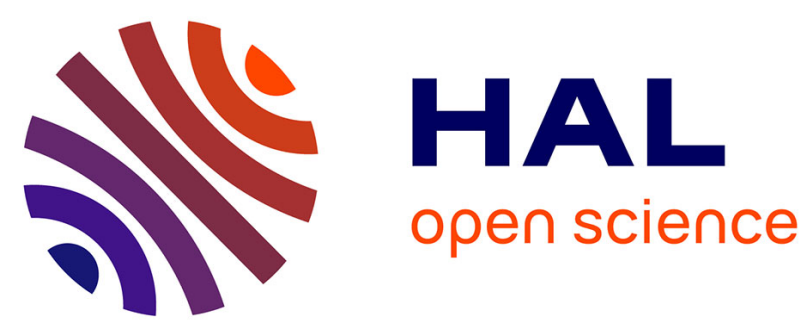

\title{
Modalités de recrutement des sujets dans la recherche en pédiatrie: étude prospective multicentrique
}

Florentia Kaguelidou, C. Iliescu, Philippe Amiel, A. Sieurin, Jean-Christophe

Roze, C. Brandt, M.-P. Cossevin, C. Gaultier, E. Jacqz-Aigrain, Corinne

Alberti

\section{To cite this version:}

Florentia Kaguelidou, C. Iliescu, Philippe Amiel, A. Sieurin, Jean-Christophe Roze, et al.. Modalités de recrutement des sujets dans la recherche en pédiatrie: étude prospective multicentrique. Epidemiology and Public Health = Revue d'Epidémiologie et de Santé Publique, 2008, 56 (S2), pp.67-68. 10.1016/j.respe.2008.03.064 . hal-00870070

\section{HAL Id: hal-00870070 https://hal.science/hal-00870070}

Submitted on 4 Oct 2013

HAL is a multi-disciplinary open access archive for the deposit and dissemination of scientific research documents, whether they are published or not. The documents may come from teaching and research institutions in France or abroad, or from public or private research centers.
L'archive ouverte pluridisciplinaire HAL, est destinée au dépôt et à la diffusion de documents scientifiques de niveau recherche, publiés ou non, émanant des établissements d'enseignement et de recherche français ou étrangers, des laboratoires publics ou privés. 


\title{
Modalités de recrutement des sujets dans la recherche en pédiatrie : étude prospective multicentrique
}

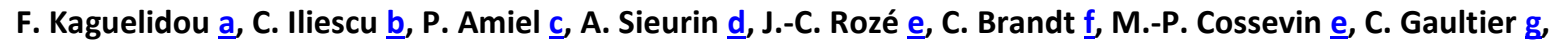 \\ E. Jacqz-Aigrain $\underline{h}, \mathbf{C}$. Alberti $\underline{\mathrm{d}}$
}

${ }^{a}$ Inserm $\mathrm{CIE5}$, réseau des $\mathrm{CIC}$ pédiatriques, CIC Robert-Debré, AP-HP, France

${ }^{\mathrm{b}} \mathrm{CIC}$ de Lille, France

${ }^{c}$ IGR, Paris, France

${ }^{d}$ Inserm CIE5, AP-HP, France

${ }^{\mathrm{e}}$ Réseau des $\mathrm{CIC}$ pédiatriques, $\mathrm{CIC}$ de Nantes, France

${ }^{f}$ Réseau des $\mathrm{CIC}$ pédiatriques, $\mathrm{CIC}$ de Strasbourg, France

${ }^{\mathrm{g}}$ DRCT, Inserm, France

${ }^{\mathrm{h}}$ Réseau des $\mathrm{CIC}$ pédiatriques, CIC Robert-Debré, AP-HP, France

Contexte. - Une enquête qualitative exploratoire a montré que le nombre de patients éligibles et sollicités dans les essais en pédiatrie était peu objectivé ainsi que les refus.

Objectif. - Estimer le nombre de refus de participation des familles dans les essais en pédiatrie et lier le taux de refus aux caractéristiques protocole, investigateur et patients.

Matériel et méthodes. - Étude prospective multicentrique inter-CIC (réseau pédiatrique) d'une cohorte de protocoles. Pour chaque sollicitation à participer, des fiches patient, investigateur et protocole étaient remplies.

Résultats. - L'étude a été réalisée de décembre 2005 à septembre 2007 sur quatre centres et a inclus 45 protocoles : 32 à promotion industrielle, 36 multicentriques, 19 essais cliniques, 33 avec prises de sang et six avec examens invasifs, 26 avec des déplacements spécifiques et 14 des hospitalisations supplémentaires. Sur ces protocoles, 170 investigateurs étaient référencés comme recruteurs et 86 (51\%) ont répondu au questionnaire : âge médian 42 ans, sex-ratio de 1, 13 sont investigateurs principal, 32 responsables pour le CIC et 50 investigateurs associés, 20 percevaient une rétribution versée au service dans $80 \%$ des cas. La charge de travail médiane par investigateur était d'une heure par inclusion et 67 (78\%) bénéficiaient d'une aide d'une TEC. Au total, 1022 sollicitations ont été réalisées sur 36 protocoles (neuf protocoles n'ayant eu aucune sollicitation) et 334 refus (33\%) ont été enregistrés soit une médiane de $12 \%$ (Q1Q3 : 0-28 \%) de refus par protocole. Parmi les 36 protocoles, 16 n'ont enregistré aucun refus, représentant 147 sollicitations et les 20 autres protocoles ont eu un taux moyen de $38 \%$ de refus. L'analyse explicative est en cours.

Conclusion. - Le taux de refus de $12 \%$ n'est pas différent de celui des essais adultes et semble dépendant du type d'étude. L'absence de sollicitation concerne $20 \%$ des études. 